\title{
CCD Photometry of Two Asteroids (895) Helio and (165) Loreley
}

\author{
H.-S. Woo ${ }^{1}$, S.-L. Kim², M.-Y. Chun ${ }^{2}$, M.-G. Park ${ }^{1}$ \\ ${ }^{1}$ Dept. of Astronomy and Atmospheric Sciences, Kyungpook National \\ University, Taegu, 702-701, Korea \\ ${ }^{2}$ Korea Astronomy Observatory, Taejon, 305-348, Korea
}

\begin{abstract}
We have presented photometric results for two asteroids (895) Helio and (165) Loreley. The observations were performed from 2000 Oct. to $2001 \mathrm{Jan}$. using the $61 \mathrm{~cm}$ telescope installed at Sobaeksan Optical Astronomy Observatory in Korea.
\end{abstract}

The asteroid (895) Helio was classified as FCB type and (165) Loreley as CD type (Tholen 1989). Their diameters are known to be $147 \mathrm{~km}$ for Helio and $160 \mathrm{~km}$ for Loreley (Clifford 1988).

Our observations were carried out in the V and $\mathrm{R}$ bands on Oct. 30, Nov. 2, Nov. 30, Dec. 1 and Dec. 3 for the asteroid (895) Helio. The asteroid (165) Loreley was observed intermittently for the latter three days, and on Jan. 16. The observing conditions for two asteroids are given in Table 1.

For the first two days, we chose GSC 02853-00901 as the comparison star for the asteroid (895) Helio. Another comparison star GSC 02322-00635 was used for the last three days. In order to correct the magnitude difference of the two comparison stars, we observed the first one during the last three nights. The comparison star for the asteroid (165) Loreley is in the USNO-A2.0 Catalogue, $\alpha=08^{h} 53^{m} 14.5, \delta=+19^{\circ} 15^{\prime} 07^{\prime \prime} .04$. Differential magnitudes of the asteroids were derived using the classical differential photometric method.

We estimated the rotational period of the asteroid (895) Helio to be about $27.792 \mathrm{hr}$ with a maximum amplitude of $0.16 \pm 0.01 \mathrm{mag}$ which is quite different from Danforth's result, $9.67 \mathrm{hr}$ and $0.21 \mathrm{mag}$. We also obtained the rotational period of the asteroid (165) Loreley to be about $7.224 \mathrm{hr}(=0.301$ day), with a maximum amplitude of $0.17 \pm 0.01 \mathrm{mag}$. That was the same period as derived by Schober et al. (1988), and our lightcurve was roughly similar to theirs.

\section{References}

Clifford, J. C. 1988, in Introduction to Asteroids, William-Bell Inc. Press, p.143 Danforth, C. W. 1994, Minor Planet Bull. 21, 4

Schober, H. J., Di Martino, M., and Cellino, A. 1988, A\&A, 197, 327

Tholen, D. J. 1989, in Asteroids II, eds. R. P. Binzel, T. Gehrels, M. S. , Matthews (University of Arizona Press) p.1139 
Table 1. Observing conditions for the asteroid (895) Helio and (165) Loreley.

\begin{tabular}{cccccc}
\hline \hline Object & Date(UT) & $\left.{ }^{\mathrm{r}} \mathrm{(AU}\right)$ & ${ }^{\ddagger} \Delta(\mathrm{AU})$ & $\alpha(2000)$ & $\delta(2000)$ \\
\hline (895) Helio & Oct. 30 & 2.816 & 1.908 & $02^{h} 43^{m} 53^{s} .6$ & $+42^{\circ} 34^{\prime} 17^{\prime \prime}$ \\
(895) Helio & Nov. 02 & 2.813 & 1.896 & $02^{h} 42^{m} 07^{s} .4$ & $+42^{\circ} 09^{\prime} 58^{\prime \prime}$ \\
(895) Helio & Nov. 30 & 2.789 & 1.897 & $02^{h} 19^{m} 01^{s} .2$ & $+36^{\circ} 38^{\prime} 01^{\prime \prime}$ \\
(895) Helio & Dec. 01 & 2.788 & 1.902 & $02^{h} 18^{m} 30^{s} .9$ & $+36^{\circ} 24^{\prime} 04^{\prime \prime}$ \\
(895) Helio & Dec. 03 & 2.786 & 1.910 & $02^{h} 17^{m} 35^{s} 3$ & $+35^{\circ} 56^{\prime} 06^{\prime \prime}$ \\
(165) Loreley & Nov. 30 & 3.380 & 2.807 & $08^{h} 53^{m} 13.6$ & $+19^{\circ} 19^{\prime} 01^{\prime \prime}$ \\
(165) Loreley & Dec. 01 & 3.380 & 2.794 & $08^{h} 53^{m} 12.1$ & $+19^{\circ} 17^{\prime} 24^{\prime \prime}$ \\
(165) Loreley & Dec. 03 & 3.380 & 2.768 & $08^{h} 53^{m} 05^{s} 3$ & $+19^{\circ} 14^{\prime} 22^{\prime \prime}$ \\
(165) Loreley & *Jan. 16 & 3.377 & 2.403 & $08^{h} 29^{m} 42.0$ & $+19^{\circ} 04^{\prime} 39^{\prime \prime}$ \\
\hline
\end{tabular}

$\dagger:$ distance between the Sun and the Asteroid, $\ddagger$ : distance between the Earth and the Asteroid, * : data in 2001.
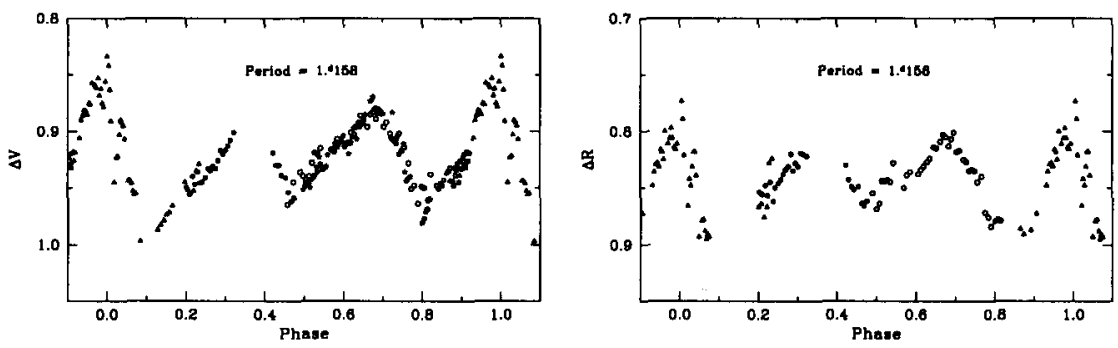

Figure 1. Phase diagram for the asteroid (895) Helio (Epoch : H.J. $D=2451848.100$ ). Left is $\mathrm{V}$ band; right is $\mathrm{R}$ band. The symbol is different for each day.

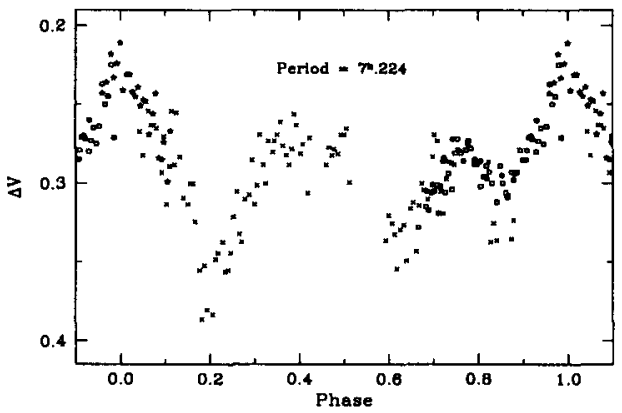

Figure 2. Phase diagram for the asteroid (165) Loreley (Epoch : H.J.D $=2451880.3087$ ). 